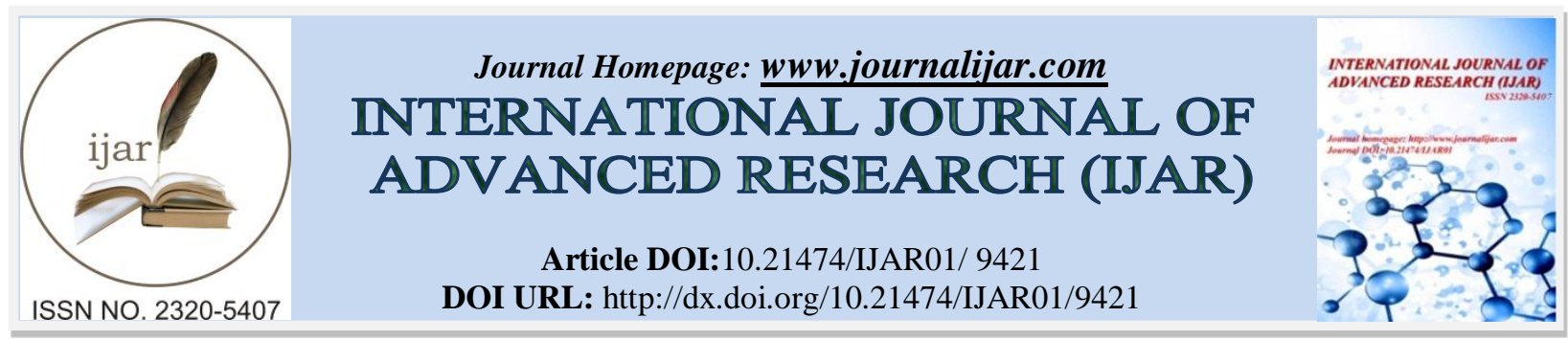

RESEARCH ARTICLE

\title{
STUDY ON HYDROGENATED CARBON ATOM CHAINS WITH NEGATIVE DIFFERENTIAL RESISTANCE EFFECT.
}

Ji-Mei Shen ${ }^{1}$, Jing Liu ${ }^{1}$ and Li-Ping Zhou ${ }^{2}$.

1. Nanjing Normal University Taizhou College, Taizhou, Jiangsu 225300, China.

2. Department of Physics, Soochow University, Suzhou 215006, China.

\section{Manuscript Info}

Manuscript History

Received: 22 May 2019

Final Accepted: 24 June 2019

Published: July 2019

Key words:

Carbon chain; Negative differential resistance; First-principles; Density functional theory;Non equilibrium Green's function.

\begin{abstract}
Using first-principles calculations combined with a nonequilibrium Green's function method, we investigate the transport properties of four carbon chains connected to armchair carbon nanotubes.At very low bias voltage, a strong negative differential resistance effect is present.Moreover, Such effect can be modified by using different radicals in the terminal of carbon chain. This paper realizes that not only "odd" carbon chains can induce negative differential resistance effect, which indicate potential applications in nanomolecular-electronics.
\end{abstract}

Copy Right, IJAR, 2019,. All rights reserved.

\section{Introduction:}

Molecular electronics is the use of molecules as the primary building block for electronic circuitry.Because of significantly decreasing device size, it would be a useful candidate for the traditional Si-based electronics. Many experimental and theoretical works demonstrated that molecule systems could be a new technological platform for electronic devices ${ }^{[1-15]}$. Recently, Yuzvinsky et al. ${ }^{[16]}$ and Chuvilin et al. ${ }^{[17]}$ show that carbon chains could be realized through grapheme nanoribbons and carbon nanotubes.several researches ${ }^{[18-24]}$ reveal that some carbon-chain based devices have negative differential resistance (NDR) effect.NDR effect is characterized by the decreases in current as the bias increases in some specific bias range.Berg et al. ${ }^{[25]}$ report that the NDR effect is valuable and practical only under low bias.Unfortunately, This effect only occurs if the number of carbon chain atoms on both side of the barrier is all odd ${ }^{[20,23]}$ Moreover, it is difficult to prepare such "odd" molecules ${ }^{[20]}$.So, the probability of the NDR effect for molecular device under low bais is only $1 / 2 \times 1 / 2$.

In order to remove the restriction of "odd" chain, we attempt to induce the molecular NDR effect at low bias by the hydrogenating terminal carbon atom in this paper.Using first-principles calculations combined with nonequilibrium Green's function (NEGF) method and the density functional theory (DFT), we investigate the transport properties of four carbon chains connected to armchair carbon nanotubes. We find that the molecular NDR effect at low bias is absent when the terminal radical of carbon chain is $-\mathrm{CH}$ and present when that is $-\mathrm{CH}_{2}$ and $-\mathrm{CH}_{3}$. Generalize to the case of "odd" carbon chains, according to the Octet rule ${ }^{[20]}$, the molecular NDR effect at low bias will be absent when the terminal radical of carbon chain is $-\mathrm{CH}_{3}$ and present when that is $-\mathrm{CH}$ and $-\mathrm{CH}_{2}$. Therefore, the probability of the molecular NDR effect at low bias for molecular devices proposed by us increases to $2 / 3 \times 2 / 3$. This probability will further increase when scanning tunneling microscopy (STM) or atomic force microscopy (AFM) ${ }^{\text {[26- }}$ ${ }^{28]}$ is used to help hydrogenated reaction because the demanded radical can be obtained if the amount of hydrogen atom adsorbed on probe and the contact time between the probe and the carbon chain are selectively controlled. 


\section{Computational details}

Fig.1 shows the molecular transport systems (TSs).Two carbon chains with four atoms contact the left and right leads of $(6,6)$ carbon nanotube, respectively.These three TSs are similar except different terminal radical in the carbon chains. From Fig.1(a) to 1(c), the terminal radical is $-\mathrm{CH},-\mathrm{CH}_{2}$ and $-\mathrm{CH}_{3}$, respectively.In the following, we call these three TSs from Fig.1(a) to 1(c) are $-\mathrm{CH}$ terminal, $-\mathrm{CH}_{2}$ terminal and $-\mathrm{CH}_{3}$ terminal, respectively. The whole TS contains three parts: the left and right leads, and the scattering region. The left and right leads are composed of $(6,6)$ carbon nanotube with four atomic layers. The carbon nanotube contacting left (right) lead in the scattering region contains five (six) atomic layers. The edge of carbon nanotube in the scattering region is hydrogenated.

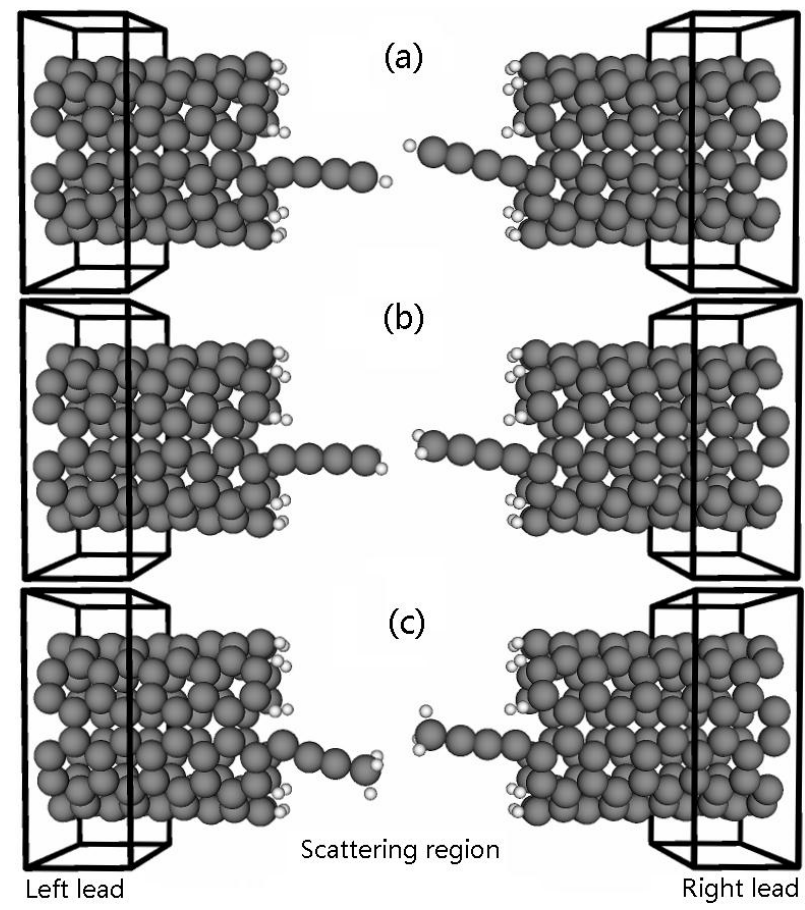

Fig.1.(Color online) Schematic illustrations of the transport systems of two capped $(6,6)$ carbon nanotubes sandwiching two

carbon chains.(a), (b) and (c) are $-\mathrm{CH},-\mathrm{CH}_{2}$ and $-\mathrm{CH}_{3}$ terminal, respectively.Color code: $\mathrm{C}$ atom (black) and $\mathrm{H}$ atom (grey).

Our calculations are performed by the Atomistix ToolKit(ATK) package, which combines the NEGF and the $\mathrm{DFT}^{[29-32]}$.A $1 \times 1 \times 50 \mathrm{k}$-points sampling is used through out the calculation and The cutoff energy is set to $250 \mathrm{Ry}$.For all $\mathrm{C}$ and $\mathrm{H}$ atoms, we choose a single- $\zeta$ plus polarization basis. The local density approximation with the Perdew and Zunger form is adopted for the exchange-correlation functional ${ }^{[33]}$. The formula(1) is used to describe the current through the scattering region,

$$
I=\frac{2 e}{h} \int_{-\infty}^{+\infty} T\left(V_{b}, E\right)\left[f_{L}\left(E-\mu_{L}\right)-f_{R}\left(E-\mu_{r}\right)\right] d E
$$

where $h$ the plank constant and e is the electron charge. $f_{L(R)}$ is the Fermi-Dirac distribution for left (right) lead, $\mu_{\mathrm{L}(\mathrm{R})}$ is the corresponding electrochemical potential. $T\left(\mathrm{~V}_{\mathrm{b}}, \mathrm{E}\right)$ is transmission coefficient,

$$
T\left(V_{b}, E\right)=\operatorname{Tr}\left[\Gamma_{L}\left(V_{b}, E\right) G\left(V_{b}, E\right) \Gamma_{R}\left(V_{b}, E\right) G^{\dagger}\left(V_{b}, E\right)\right]
$$

\section{Results and discussions:}

Fig. 2 shows the current-voltage $(I-V)$ curve of $-\mathrm{CH}$ terminal, $-\mathrm{CH}_{2}$ terminal and $-\mathrm{CH}_{3}$ terminal, respectively.In Fig.2, the molecular NDR effect at low bias is present for $-\mathrm{CH}_{2}$ terminal and $-\mathrm{CH}_{3}$ terminal while absent for $-\mathrm{CH}$ terminal.The current for $-\mathrm{CH}$ terminal is almost inhibited all over bias region.The peak position of bias $\left(\mathrm{V}_{\text {peak }}\right)$ corresponding to the peak position of current $\left(\mathrm{I}_{\text {peak }}\right)$ for $-\mathrm{CH}_{2}\left(-\mathrm{CH}_{3}\right)$ terminal is $0.20 \mathrm{~V}(0.34 \mathrm{~V})$ while the valley 
position of bias $\left(\mathrm{V}_{\text {valley }}\right)$ corresponding to the valley position of current $\left(\mathrm{I}_{\text {valley }}\right)$ for $-\mathrm{CH}_{2}\left(-\mathrm{CH}_{3}\right)$ terminal is $1.16 \mathrm{~V}$ $(1.42 \mathrm{~V})$. .I should be mentioned here that the molecular NDR effect at low bias also present in carbon atom chains sandwiched between two graphene nanoribbon ${ }^{[34-36]}$.The peak-to-valley ratio (the ratio of $\mathrm{I}_{\text {peak }}$ to $\mathrm{I}_{\text {valley }}$ ) for $-\mathrm{CH}_{2}$ ($\mathrm{CH}_{3}$ ) terminal is as large as $679 \%$ (416\%). The different $\mathrm{V}_{\text {peak }}$ for $-\mathrm{CH}_{2}$ and $-\mathrm{CH}_{3}$ terminal also means that the molecular NDR effect at low bias can be adjusted by adjusting the modified radical, which indicate potential applications in the future.

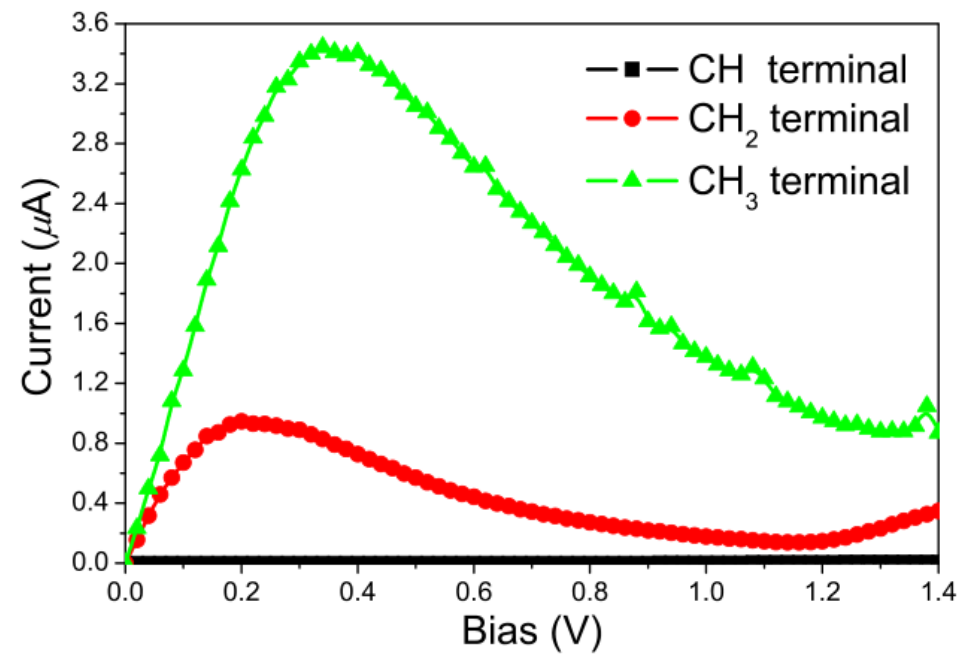

Fig.2.(Color online) The current-bias curve of the transport systems.

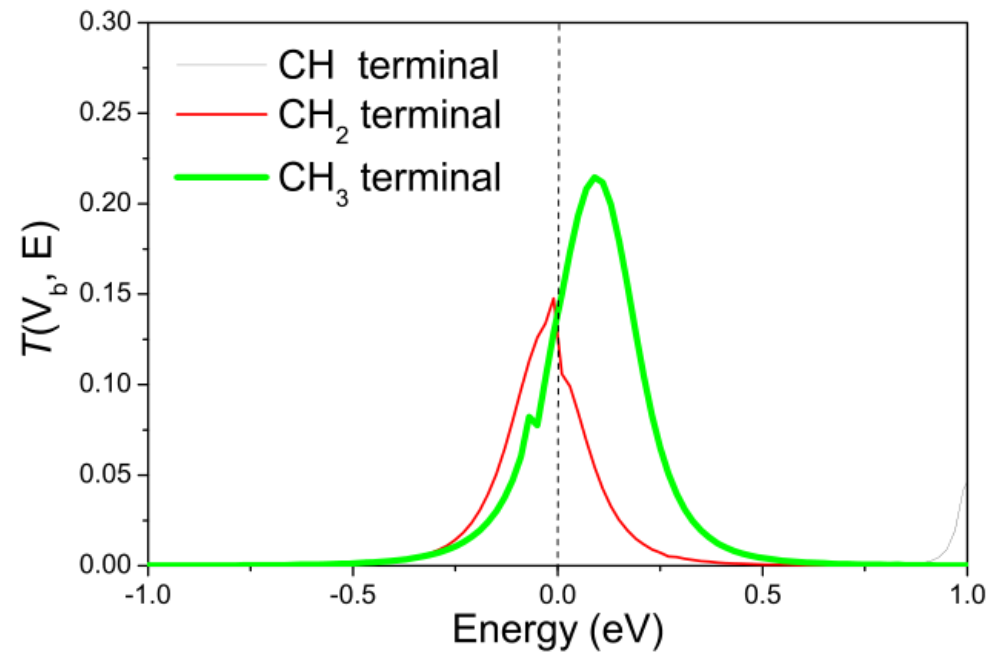

Fig.3.(Color online) Equilibrium $T\left(\mathrm{~V}_{\mathrm{b}}, \mathrm{E}\right)$ spectrum of the transport systems. The Fermi level is indicated by a dashed line.

Figs.3 shows $\mathrm{T}(\mathrm{Vb}, \mathrm{E})$ spectrum at zero bias for TSs.One can see a broad and high $T\left(\mathrm{~V}_{\mathrm{b}}, \mathrm{E}\right)$ peak around the Fermi level for $-\mathrm{CH}_{2}$ and $-\mathrm{CH}_{3}$ terminal while no peaks for $-\mathrm{CH}$ terminal as shown in the Figs..In the following, we discard - $\mathrm{CH}$ terminal because of no NDR effect.Spatial distributions of molecular orbital (MO) levels around the Fermi level are shown in Fig.4, It is cleare that these MO levels are the highest occupied MO (HOMO) and HOMO1 for $-\mathrm{CH}_{2}$ terminal while are HOMO-1, $\mathrm{HOMO}$, the lowest unoccupied MO (LUMO), LUMO+1 for $-\mathrm{CH}_{3}$ terminal.The HOMO and HOMO- 1 of $-\mathrm{CH}_{2}$ terminal tightly closes to each other in energy space and respectively locates on the left and right carbon chains in the scattering region, which is very beneficial for the resonance transmission between two vacuum separating carbon chains. The similar case is also present for the $-\mathrm{CH}_{3}$ terminal.For these reasons, the zero bias $T\left(\mathrm{~V}_{\mathrm{b}}, \mathrm{E}\right)$ peak around the Fermi level for $-\mathrm{CH}_{2}$ and $-\mathrm{CH}_{3}$ terminal is 
contributed by these $\mathrm{MO}$ levels around Fermi level.For the $-\mathrm{CH}_{3}$ terminal, the resonance transmission not only presents between LUMO and LUMO+1 but also in the delocalized HOMO-1 and HOMO.Consequently, the $T\left(\mathrm{~V}_{\mathrm{b}}\right.$, E) peak around the Fermi level in $-\mathrm{CH}_{3}$ terminal is higher than that in $-\mathrm{CH}_{2}$ terminal.

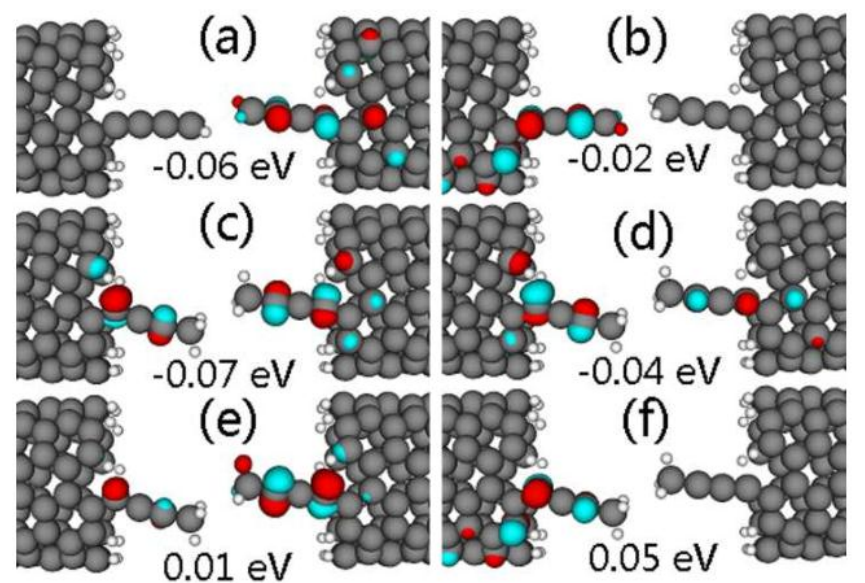

Fig.4.(Color online) spatial distributions of MO levels closed to the Fermi level at zero bias.(a), (b) are respectively HOMO-1,

HOMO for $-\mathrm{CH}_{2}$ terminal while (c), (d), (e), (f) are respectively HOMO-1, HOMO, LUMO, $\mathrm{LUMO}+1$ for $-\mathrm{CH}_{3}$ terminal.
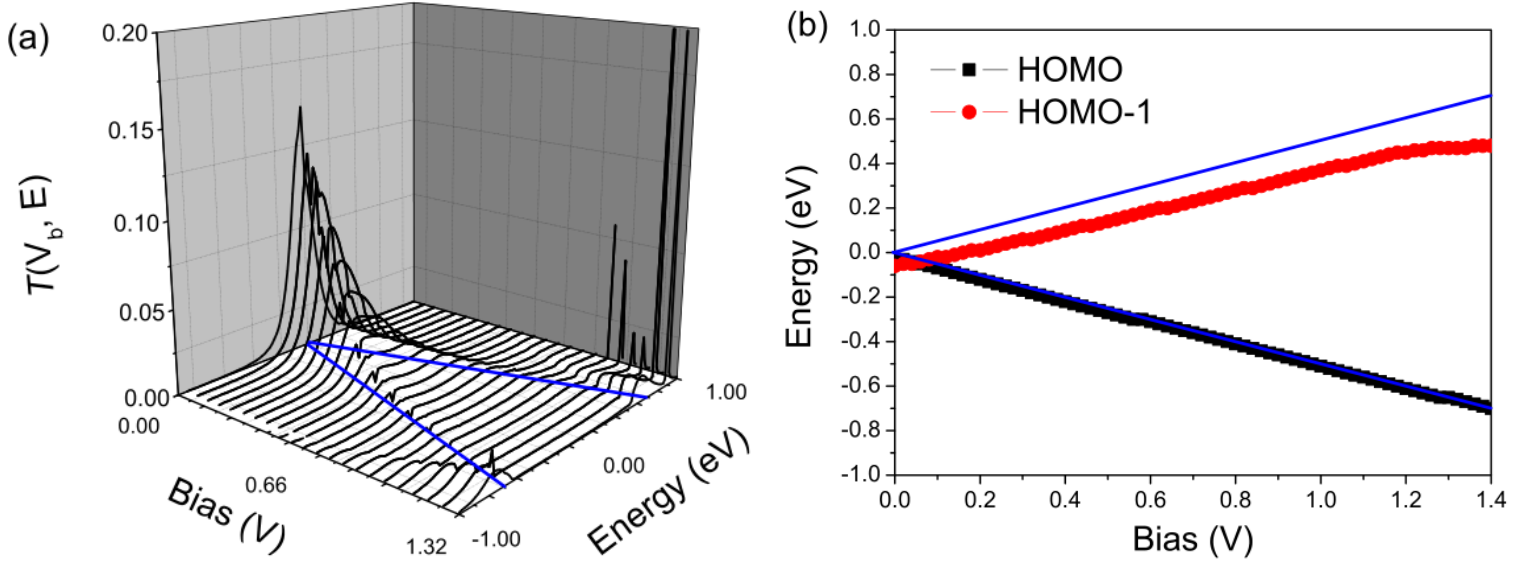

Fig.5.(Color online) (a) and (b) are bias-dependent $T\left(\mathrm{~V}_{\mathrm{b}}\right.$, E) spectra and MO levels for $-\mathrm{CH}_{2}$ terminal, respectively.The energy

region between two blue solid line is transport window.

To penetrate the mechanism which results in the NDR effect, we study the bias-dependent $T\left(\mathrm{~V}_{\mathrm{b}}, \mathrm{E}\right)$ spectra and MO levels around the Fermi level below. As represent, we only analyze the transport properties of $-\mathrm{CH}_{2}$ terminal because the mechanism resulting in the NDR effect for $-\mathrm{CH}_{3}$ terminal is similar with that for $-\mathrm{CH}_{2}$ terminal except that the bias voltage will transfer the delocalized HOMO-1 and HOMO to localized orbital ${ }^{[21]}$ in the $-\mathrm{CH}_{3}$ terminal.The bias-dependent $T\left(\mathrm{~V}_{\mathrm{b}}\right.$, E) spectra for the $-\mathrm{CH}_{2}$ terminal is shown in Fig.5(a).From the Fig., one can clearly see that the $T\left(\mathrm{~V}_{\mathrm{b}}, \mathrm{E}\right)$ peak around the Fermi level gradually becomes broad and low and eventually disappears as the bias increases. These phenomena can be explained by the bias-dependent MO levels shown in Fig.5(b).As mentioned above, the HOMO (HOMO-1) is localized in the left (right) carbon chains. When the bias $+\mathrm{V}_{\mathrm{b}} / 2$ is applied to the left lead, the electrochemical potential in the left lead is shifted down by $\mathrm{V}_{\mathrm{b}} / 2$, and the potential in the right lead is shifted up by $\mathrm{V}_{\mathrm{b}} / 2$ as the bias $-\mathrm{V}_{\mathrm{b}} / 2$ is applied to the right lead.The HOMO (HOMO-1) shifts down (up) rough following the left (right) lead because these two carbon chains are separated by vacuum barrier.It is clear that the HOMO and HOMO-1 indeed leaves each other when the bias increases, as shown in Fig.5(b).At zero bias, as mentioned above, the $T\left(\mathrm{~V}_{\mathrm{b}}, \mathrm{E}\right)$ peak around the Fermi level originate from the resonance transmission between 
HOMO and HOMO-1 because the energy difference between them is negligibly small.Due to the effect of coherent tunneling ${ }^{[38]}, T\left(\mathrm{~V}_{\mathrm{b}}, \mathrm{E}\right)$ peak around the Fermi level will be still present but becomes low and broad when the HOMO and HOMO-1 leaves each other in energy sapce.This $T\left(\mathrm{~V}_{\mathrm{b}}, \mathrm{E}\right)$ peak will eventually disappear when the energy difference of HOMO and HOMO-1 is adequate large.From zero bias to $\mathrm{V}_{\text {peak }}$, the increase of transport window predominates transport characteristics and promotes the increase of current.From $V_{\text {peak }}$ to $V_{\text {valley, the energy }}$ difference of HOMO and HOMO-1 predominates transport characteristics and reduces the current.Hence, the NDR effect at low bias is present.

\section{Conclusions:-}

In summary, using a first-principles calculations which combine NEGF with DFT, we realize one-dimensional NDR effect by four carbon atom chains, and the possibility of the NDR effect at low bias is increased.Our calculations also reveal that one can adjust the molecular NDR effect at low bias by adjusting the modified radicals, which indicate potential molecular-electronics applications.

\section{References:-}

1. A. Aviram and M. Ratner, Chem. Phys. Lett. 29 (1974) 277.

2. J. Chen et al., Science 286 (1999) 1550.

3. M. Reed et al., Appl. Phys. Lett. 78 (2001) 3735.

4. J. Taylor, H. Guo and J. Wang, Phys. Rev. B 63 (2001) 121104.

5. I. I. Oleynik et al., Phys. Rev. Lett. 96 (2006) 096803.

6. J. B. Pan et al., Appl. Phys. Lett. 98 (2011) 092102.

7. H. Y. He et al., J. Phys. Chem. C 113 (2009) 1575.

8. Y. Min et al., Int. J. Mod. Phys. B 27 (2013) 1350081.

9. M. Q. Long, K. Q. Chen and L. L. Wang, Appl. Phys. Lett. 91 (2007) 233512.

10. S. J. van der Molen et al., Nano Lett. 9 (2009) 76.

11. Z. C. Wang et al., Appl. Phys. Lett. 93 (2008) 152106.

12. Y. Ren et al., Appl. Phys. Lett. 94 (2009) 183506.

13. Y. Min et al., Phys. Lett. A 378 (2014) 1170.

14. R. P. Andres et al., Science 272 (1996) 1323.

15. Y. Min et al., Appl. Phys. A 118 (2015) 367.

16. T. Yuzvinsky et al., Nano Lett. 6, 2718 (2006).

17. A. Chuvilin et al., New J. Phys. 11 (2009) 083019.

18. K. H. Khoo et al., Nano Lett. 8 (2008) 2900.

19. Z. Zhang et al., Adv. Funct. Mater. 23 (2013) 2765.

20. A. Mahmoud and P. Lugli, Appl. Phys. Lett. 103 (2013) 033506.

21. Z. H. Zhang et al., Carbon 51 (2013) 313.

22. H. Wan, Y. Xu and G. Zhou, J. Chem. Phys. 136 (2012) 184704.

23. Q. Wu et al., Chem. Phys. Lett. 605-606 (2014) 62.

24. X. Z. Wu et al., Physica E 45 (2012) 82.

25. J. Berg, S. Bengtsson and P. Lundgren, Solid State Electron. 44 (2000) 2247.

26. K. Zhang et al., Nat. Commun. 3 (2012) 1194.

27. S. Masubuchi et al., Appl. Phys. Lett. 94 (2009) 082107.

28. L. S. Weng et al., Appl. Phys. Lett. 93 (2008) 093107.

29. Atomistix ToolKit, Quantumwise A/S (www.quantumwise.com).

30. M. Brandbyge et al., Phys. Rev. B 65 (2002) 165401.

31. J. M. Soler et al., J. Phys.: Condens. Matter 14 (2002) 2745.

32. Y. Min et al., Phys. Lett. A 379 (2015) 2637.

33. J. P. Perdew and A. Zunger, Phys. Rev. B 23 (1981) 5048.

34. Y. H. Zhou et al., Carbon 95 (2015) 503.

35. X. F. Yang et al., Org. Electron. 55 (2018) 170.

36. A. L. Yao et al., Appl. Nanosci. 9(2019) 143. 